This is the final peer-reviewed accepted manuscript of:

Biagi, Stefano, Giovanni Cupini, e Elvira Mascolo. «Regularity of quasi-minimizers for nonuniformly elliptic integrals». Journal of Mathematical Analysis and Applications 485, n. 2 (2020): 123838.

\begin{tabular}{|l}
$\begin{array}{l}\text { The final published version is available online at: } \\
\text { https://doi.org/10.1016/j.jmaa.2019.123838 }\end{array}$ \\
\hline
\end{tabular}

Rights / License:

The terms and conditions for the reuse of this version of the manuscript are specified in the publishing policy. For all terms of use and more information see the publisher's website.

This item was downloaded from IRIS Università di Bologna (https://cris.unibo.it/)

When citing, please refer to the published version. 


\title{
REGULARITY OF QUASI-MINIMIZERS FOR NON-UNIFORMLY ELLIPTIC INTEGRALS
}

\author{
STEFANO BIAGI, GIOVANNI CUPINI, ELVIRA MASCOLO
}

\begin{abstract}
In this paper we consider a class of non-uniformly elliptic integral functionals and we prove the local boundedness of the quasi-minimizers. Our approach is based on a suitable adaptation of the celebrated De Giorgi proof, and it relies on an appropriate Caccioppoli-type inequality.
\end{abstract}

\section{INTRODUCTION}

In the present paper we shall be concerned with a class of non-uniformly elliptic functionals $\mathcal{F}$ of the Calculus of Variations taking the following form

$$
\mathcal{F}: W_{\text {loc }}^{1,1}(\Omega) \rightarrow[0, \infty], \quad \mathcal{F}(u):=\int_{\Omega} f(x, u, \nabla u) \mathrm{d} x,
$$

where $\Omega \subseteq \mathbb{R}^{n}$ is a bounded open set and $f$ is a real-valued Carathéodory function on $\Omega \times \mathbb{R} \times \mathbb{R}^{n}$ satisfying the non-uniform growth assumption

$$
\lambda(x)|\xi|^{p} \leq f(x, u, \xi) \leq \mu(x)\left(|\xi|^{p}+|u|^{q}\right)+a(x) .
$$

In the above (1.2), $p, q$ are positive real numbers such that $q \geq p>1$ and $\lambda, \mu, a$ are non-negative measurable functions fulfilling suitable integrability assumptions (see, precisely, Theorem 1.1 below and Theorem 2.2 in Section 2).

Our main aim is to prove the (local) boundedness of any quasi-minimizer of $\mathcal{F}$. By quasi-minimizer of $\mathcal{F}$ we mean a function $u \in W_{\mathrm{loc}}^{1,1}(\Omega)$ with $f(x, u, \nabla u) \in L_{\mathrm{loc}}^{1}(\Omega)$ and for which there exists some $Q \geq 1$ such that

$$
\int_{\operatorname{supp} \varphi} f(x, u, \nabla u) \mathrm{d} x \leq Q \int_{\operatorname{supp} \varphi} f(x, u+\varphi, \nabla(u+\varphi)) \mathrm{d} x,
$$

for every $\varphi \in W^{1,1}(\Omega)$ with $\operatorname{supp} \varphi \Subset \Omega$ (see also Definition 2.1 in Section 2).

As is well-known, the development of the theory of regularity for single equations and scalar integrals is strictly connected with the pioneering result established by De Giorgi [8] in 1957. Indeed, De Giorgi considered a linear elliptic equation (with associated quadratic functional) of the form

$$
\sum_{i, j=1}^{n} \frac{\partial}{\partial x_{i}}\left(a_{i j}(x) \frac{\partial u}{\partial_{x_{j}}}(x)\right)=0 \quad\left(x \in \Omega \subseteq \mathbb{R}^{n}\right)
$$

2010 Mathematics Subject Classification. Primary: 49N60; Secondary: 35J60.

Key words and phrases. Non-uniformly elliptic functionals, regularity of quasi-minimizers, local boundedness, Caccioppoli-type inequality.

Acknowledgement: We thank the anonymous Referee for his/her precious suggestions. We are members of Gruppo Nazionale per l'Analisi Matematica, la Probabilità e le loro Applicazioni (GNAMPA) of the Istituto Nazionale di Alta Matematica (INdAM). 
where the functions $a_{i j}$ 's are measurable and bounded in $\Omega$, and the $n \times n$ matrix $A(x)=\left(a_{i j}(x)\right)_{i j}$ is symmetric (for all $x \in \Omega$ ). Assuming that, for some $\lambda>0$,

$$
\sum_{i, j=1}^{n} a_{i j}(x) \xi_{i} \xi_{j} \geq \lambda|\xi|^{2} \quad \text { for all } \xi \in \mathbb{R}^{n} \text {, a.e. } x \in \Omega,
$$

De Giorgi proved that any weak solution $u$ of (1.4) is locally bounded and, further, locally Hölder continuous. One of the crucial points in the De Giorgi's proof is the following Caccioppoli inequality on the super-level sets of $u$ :

$$
\int_{A_{\beta, \rho}}|\nabla u|^{2} \mathrm{~d} x \leq \frac{c}{(R-\rho)^{p}} \int_{A_{\beta, R}}(u-\beta)^{2} \mathrm{~d} x \quad \text { (valid for all } 0<\rho<R \text { ), }
$$

where $R>0$ is such that $B\left(x_{0}, R\right) \Subset \Omega$ and, for any $\beta \in \mathbb{R}$, where

$$
A_{\beta, R}:=\left\{x \in B\left(x_{0}, R\right): u(x)>\beta\right\} .
$$

It is out of doubts that De Giorgi's regularity result [8], together with the subsequent results by Moser [20] and Stampacchia [22], produced a decisive progress in the theory of regularity. Starting from these seminal works, several improvements (regarding the scalar case) have been achieved: for example, Giaquinta and Giusti in the series of papers $[11,12,13]$ proved the Hölder regularity for the quasi-minimizers of a class of non-differentiable scalar functionals as in (1.1) assuming that the integrand function $f$ satisfies the following $p$-growth conditions:

$$
|\nabla u|^{p} \leq f(x, u, \nabla u) \leq C(1+|\nabla u|)^{p} \quad(\text { with } p>1) .
$$

We refer the reader to [14] for a comprehensive treatment of this topic.

In [23], Trudinger started the study of the regularity for non-uniformly elliptic equations. More precisely, he considered equations as in (1.4) assuming that

$$
\lambda(x)|\xi|^{2} \leq \sum_{i, j=1}^{n} a_{i j}(x) \xi_{i} \xi_{j} \leq n^{2} \mu(x)|\xi|^{2}
$$

for a.e. $x \in \Omega$ and any $\xi \in \mathbb{R}^{n}$. Here, $\lambda(x)$ is the minimum eigenvalue of the symmetric matrix $A(x)=\left(a_{i j}(x)\right)$ and $\mu:=\sup _{i j}\left|a_{i j}\right|$. The main novelty in Trudinger's result (which also highlights the non-uniformity of (1.6)) relies in the assumption that

$$
\lambda^{-1} \in L_{\mathrm{loc}}^{r}(\Omega) \quad \text { and } \quad \mu_{1}=\lambda^{-1} \mu^{2} \in L_{\mathrm{loc}}^{\sigma}(\Omega) \quad \text { with } \frac{1}{r}+\frac{1}{\sigma}<\frac{2}{n},
$$

hence the ratio $\mu / \lambda$ is not necessarily bounded in $\Omega$. Under conditions (1.6) and (1.7), Trudinger proved that any weak solution of (1.4) is locally bounded in $\Omega$.

As is well-known, the equation (1.4) is usually called degenerate when the $a_{i j}$ 's fulfill (1.6) but $\lambda^{-1} \notin L^{\infty}(\Omega)$, whereas it is called singular when $\mu \notin L^{\infty}(\Omega)$. It is worth emphasizing that the theory of regularity for (1.4) under assumption (1.6) is significantly complicated by the $x$-dependence of $\lambda$ and $\mu$; in fact the problem of finding optimal additional conditions on $\lambda$ and $\mu$ which guarantee the Hölder continuity of the solutions of (1.4) is still an open problem.

The result by Trudinger previously described was extended in many settings and directions throughout the years: firstly by Trudinger himself in [24], where more general equations are considered; later on, among several other authors, by Fabes, Kenig and Serapioni in [10]. In this paper, the authors establish the local regularity for the solutions of degenerate equations satisfying (1.6) with $\lambda=\mu$ belonging to the Muckenhoupt class (see [21]).

Clearly, the literature concerning non-uniformly elliptic problems is quite extensive, and it is beyond our scopes to give an exhaustive list of references; here, we limit ourselves to mention the most recent papers $[1,5,6,7,9,15,17,18,19,25]$, and we refer the interested reader to the bibliography therein. 
We now turn to describe the main result of this paper. As already said, we aim at proving the (local) boundedness of any quasi-minimizer of $\mathcal{F}$ in (1.1). In our context, we require the integrand function $f$ to satisfy the generalized version of Trudinger's assumptions, namely we assume that

- $f$ is a Carathédory function on $\Omega \times \mathbb{R} \times \mathbb{R}^{n}$ (where $\Omega \subseteq \mathbb{R}^{n}$ is open);

- $f(x, \cdot, \cdot)$ is convex on $\mathbb{R} \times \mathbb{R}^{n}$ for every fixed $x \in \Omega$;

- $f$ satisfies the growth condition (1.2), where $q \geq p>1$ and $\lambda, \mu, a: \Omega \rightarrow \mathbb{R}$ are non-negative measurable functions such that, for a suitable positive constant $k$, one has $\lambda(x) \leq k \mu(x)$ for a.e. $x \in \Omega$.

While we postpone to Section 2 the statement of our result in its full generality, we now give the statement in the particular case when (1.2) holds with $q=p$.

Theorem 1.1. Assume that $f$ satisfies (1.2) with $q=p$, and

$$
\lambda^{-1} \in L_{\mathrm{loc}}^{r}(\Omega), \quad \mu \in L_{\mathrm{loc}}^{\sigma}(\Omega), \quad a \in L_{\mathrm{loc}}^{\tau}(\Omega),
$$

for some $r \in[1, \infty], \sigma \in(1, \infty]$ and $\tau \in(1, \infty]$ fulfilling

$$
\frac{1}{r}+\max \left\{\frac{1}{\sigma}, \frac{1}{\tau}\right\}<\frac{p}{n} \quad \text { and } \quad r \geq \frac{1}{p-1} \quad \text { if } p \in(1,2) \text {. }
$$

If $u \in W_{\mathrm{loc}}^{1,1}(\Omega)$ is a quasi-minimizer for the functional $\mathcal{F}$ in (1.1), then $u$ is locally bounded in $\Omega$.

We refer to Theorem 2.2 for the general case $1<p \leq q$, and for a quantitative estimate of the $L^{\infty}$-norm of the quasi-minimizers. We explicitly point out that a crucial assumption in Theorem 2.2 is the following

$$
q \sigma^{\prime}<\nu^{*}, \quad \text { where } \nu=\frac{p r}{r+1},
$$

(with the convention $\nu:=p$ if $r=+\infty$ ), $\nu^{*}$ is the Sobolev exponent of $\nu$ and $\sigma^{\prime}$ is the conjugate exponent of $\sigma$. When $\lambda=1$ and $\mu \in L^{\infty}(\Omega)$, the above (1.10) gives back the classical bound $q<p^{*}$ (see, e.g., [14]).

Although being inspired by Trudinger's result, the result in Theorem 1.1 is significantly more general if compared with the previous ones: firstly, we consider quasiminimizers of integral functionals $\mathcal{F}$, so that no Euler-Lagrange equation is available; secondly, the function $f$ defining $\mathcal{F}$ is assumed to satisfy a $p$-growth condition with a general $p>1$, and an explicit dependence of $u$ is allowed. We explicitly point out that our integrability assumptions (1.8)-(1.9) on $\lambda, \mu$ and $a$ reduce to those which have been considered for less general cases: for example, when $p=2$ or when $\lambda$ and $\mu$ are positive constants (for more details see Remark 2.4).

We now spend a few words about the proof of Theorem 2.2 (of which Theorem 1.1 is a particular case). Broadly speaking, our approach is based on a suitable adaptation of De Giorgi's iterative method, which needs to take into account both the (possible) degeneracy of the 'weights' $\lambda, \mu$ and the different exponents $p, q$ in (1.2). These facts make the proof definitely more involved than in the classical cases. The key ingredients of our approach are, essentially, the following:

(1) First of all, we prove a Caccioppoli-type inequality for any quasi-minimizer $u$ of $\mathcal{F}$ : more precisely, fixed $x_{0} \in \Omega$ and a ball $B\left(x_{0}, R_{0}\right) \Subset \Omega$ we have that there exists $\Lambda>0$ such that, for every $0<\rho<R \leq R_{0}$ and every $\beta \in \mathbb{R}$ with $\beta \geq 1$, we have

$$
\begin{gathered}
\int_{A_{\beta, \rho}} f(x, u, \nabla u) \mathrm{d} x \leq \Lambda\left\{\frac{1}{(R-\rho)^{p}} \int_{A_{\beta, R}} \mu(x)\left((u-\beta)^{p}+\beta^{q}\right) \mathrm{d} x\right. \\
\left.\quad+\|a\|_{L^{\tau}\left(B\left(x_{0}, R\right)\right)} \cdot\left|A_{\beta, R}\right|^{1-\frac{1}{\tau}}\right\} .
\end{gathered}
$$


(2) With the Caccioppoli-type inequality (1.11) at hand, the second ingredient is a decay estimate of the "excess" on the superlevel sets of $u$. Fixed a suitable level $\beta \geq 1$, we define a sequence $\rho_{h}$ of radii starting from $R_{0}$ and decreasing to $\frac{R_{0}}{2}$ and another sequence $\beta_{h}$ of levels starting from $\frac{\beta}{2}$ and increasing to $\beta$. We then define the "excess" on the superlevel set as follows

$$
J_{h}:=\int_{A_{\beta_{h}, \rho_{h}}}\left(u-\beta_{h}\right)^{p \sigma^{\prime}} d x .
$$

Using the Caccioppoli-type inequality (1.11) and the Sobolev-type Embedding in Lemma 4.2 , we are able to show that

$$
J_{h+1} \leq C\left(1+\|u\|_{L^{\nu^{*}\left(B\left(x_{0}, R_{0}\right)\right)}}^{(q-p) \sigma^{\prime}}\right) \zeta^{h} J_{h}^{1+\alpha},
$$

where $C, \zeta>1$ are suitable constants depending on the data and

$$
\alpha:=\min \left\{1-\frac{q \sigma^{\prime}}{\nu^{*}}, \sigma^{\prime}\left(1-\frac{1}{\tau}-\frac{p}{\nu^{*}}\right)\right\}
$$

(3) Having established (1.13), the local (upper) boundedness of $u$ follows by a standard iteration argument: choosing $\beta>1$ large enough, and using the fact that $\alpha>0$ (which is a consequence of assumptions (1.9) and (1.10)), one gets

$$
\lim _{h \rightarrow+\infty} J_{h}=0
$$

in its turn, the above (1.14) readily implies that

$$
u \leq \beta \quad \text { a.e. in } B\left(x_{0}, R_{0} / 2\right) .
$$

Lower bounds for $u$ can be obtained by showing that $-u$ is a quasi-minimizer for a similar functional, so that $-u$ is bounded from above.

We conclude this Introduction by explicitly highlighting that our result applies to several energy integrals which are nowadays receiving great attention in the literature; this is the case, e.g., of the double phase energy integral

$$
\mathcal{F}(v)=\int_{\Omega}\left(h_{1}(x)|\nabla v(x)|^{s}+h_{2}(x)|\nabla v(x)|^{p}\right) \mathrm{d} x \quad 1<s<p,
$$

with non-negative functions $h_{1}$ and $h_{2}$. A deep study of such functionals was recently carried out under many different aspects in the framework of $p, q$-growth conditions, see e.g. $[2,3,4]$. It is straightforward to check that the integrand function

$$
f(x, \xi):=h_{1}(x)|\xi|^{s}+h_{2}(x)|\xi|^{p}
$$

fulfills the growth condition (1.2) with

$$
\lambda(x):=h_{2}(x), \quad \mu(x):=\left(h_{1}(x)+h_{2}(x)\right), \quad a(x):=h_{1}(x) .
$$

As a consequence, if we assume that

$$
h_{2}^{-1} \in L_{\mathrm{loc}}^{r}(\Omega), \quad\left(h_{1}+h_{2}\right) \in L_{\mathrm{loc}}^{\sigma}(\Omega) \quad \text { and } \quad h_{1} \in L_{\mathrm{loc}}^{\tau}(\Omega)
$$

for some $r \in[1, \infty), \sigma \in(1, \infty), \tau \in(1, \infty)$ satisfying the relations (1.9), we infer from Theorem 1.1 that any quasi-minimizer of $\mathcal{F}$ is locally bounded, without any relationship between the exponents $p$ and $s$.

Another application of Theorem 1.1 is the following (see also the more general Example 2.3 in Section 2): consider the functional

$$
\mathcal{F}(v)=\int_{\Omega} h(x)\left(|\nabla v(x)|^{p}+|u(x)|^{p}\right) \mathrm{d} x,
$$


where $p>1$ and $h$ is non-negative on $\Omega$. If we assume that

$$
h^{-1} \in L_{\mathrm{loc}}^{r}(\Omega) \quad \text { and } \quad h \in L_{\mathrm{loc}}^{\sigma}(\Omega)
$$

for some $r \in[1, \infty), \sigma \in(1, \infty)$ satisfying

$$
\frac{1}{r}+\frac{1}{\sigma}<\frac{p}{n} \quad \text { and } \quad r \geq \max \left\{1, \frac{1}{p-1}\right\}
$$

then the quasi-minimizers of $\mathcal{F}$ are locally bounded.

Plan of the paper. A short plan of the paper is now in order.

- In Section 2 we shall give the needed preliminary definitions, the statement of our result in its full generality, Theorem 2.2, together with some remarks concerning our main assumptions.

- In Section 3 we shall prove a Caccioppoli-type inequality for the quasi-minimizers of $\mathcal{F}$, which is the fundamental tool for the proof of Theorem 2.2.

- In Section 4 we shall set up the De Giorgi's iterative method, by means of which we shall be able to provide the proof of Theorem 2.2.

\section{Preliminary Definitions and statement of the main Result}

As anticipated in the Introduction, throughout this paper we shall be concerned with integral functionals of the following form

$$
\mathcal{F}: W_{\mathrm{loc}}^{1,1}(\Omega) \longrightarrow[0, \infty], \quad \mathcal{F}(u):=\int_{\Omega} f(x, u, \nabla u) \mathrm{d} x,
$$

where $\Omega \subseteq \mathbb{R}^{n}$ is a fixed open set and $f: \Omega \times \mathbb{R} \times \mathbb{R}^{n} \rightarrow \mathbb{R}$ is a Carathéodory function satisfying the following assumptions (already presented in the Introduction):

$$
f(x, \cdot, \cdot) \text { is convex on } \mathbb{R} \times \mathbb{R}^{n} \text { for every fixed } x \in \Omega \text {; }
$$

and, for every $x \in \Omega$ and every $(u, \xi) \in \mathbb{R} \times \mathbb{R}^{n}$, one has

$$
\lambda(x)|\xi|^{p} \leq f(t, u, \xi) \leq \mu(x)\left(|\xi|^{p}+|u|^{q}\right)+a(x),
$$

where $q \geq p>1$ and $a, \lambda, \mu: \Omega \rightarrow(0, \infty)$ are measurable functions such that

$$
\lambda(x) \leq k \mu(x) \text { a.e. in } \Omega,
$$

for a suitable constant $k>0$. Furthermore, we require that

$$
\lambda^{-1} \in L_{\mathrm{loc}}^{r}(\Omega), \quad \mu \in L_{\mathrm{loc}}^{\sigma}(\Omega), \quad a \in L_{\mathrm{loc}}^{\tau}(\Omega),
$$

for some $r \in[1, \infty], \sigma \in(1, \infty]$ and $\tau \in(1, \infty]$ fulfilling the relations

$$
\frac{1}{r}+\max \left\{\frac{1}{\sigma}, \frac{1}{\tau}\right\}<\frac{p}{n}, \quad \text { and } \quad r \geq \frac{1}{p-1} \quad \text { if } p \in(1,2) .
$$

Since we aim at proving the local boundedness of the quasi-minimizers of the functional $\mathcal{F}$, we remind their precise definition.

Definition 2.1. We say that a function $u \in W_{\text {loc }}^{1,1}(\Omega)$ is a quasi-minimizer of $\mathcal{F}$ in (2.1) if $x \mapsto f(x, u, \nabla u) \in L_{\mathrm{loc}}^{1}(\Omega)$ and there exists a real $Q \geq 1$ such that

$$
\int_{\operatorname{supp} \varphi} f(x, u, \nabla u) \mathrm{d} x \leq Q \int_{\operatorname{supp} \varphi} f(x, u+\varphi, \nabla(u+\varphi)) \mathrm{d} x,
$$

for every $\varphi \in W^{1,1}(\Omega)$ with supp $\varphi \Subset \Omega$. In the particular case when $(2.7)$ holds with $Q=1$, we say that $u$ is a local minimizer of $\mathcal{F}$. 
With simple computations (see e.g. Proposition 3.1 in [7]), for every open set $\Omega^{\prime}$ compactly contained in $\Omega$ we deduce that

$$
\left\|\lambda^{-1}\right\|_{L^{r}\left(\Omega^{\prime}\right)}^{-1}\|\nabla v\|_{L^{\frac{p r}{r+1}}\left(\Omega^{\prime} ; \mathbb{R}^{n}\right)}^{p} \leq \int_{\Omega^{\prime}} f(x, v, \nabla v) d x .
$$

From (2.8) and the classical Sobolev embedding theorem, we then get

$$
W^{1, \frac{p r}{r+1}}\left(\Omega^{\prime}\right) \supset W^{1, \mathcal{F}}\left(\Omega^{\prime}\right)
$$

where $W^{1, \mathcal{F}}\left(\Omega^{\prime}\right)$ denotes the set of maps $u$ with finite $\mathcal{F}$-energy on $\Omega^{\prime}$, that is,

$$
W^{1, \mathcal{F}}\left(\Omega^{\prime}\right):=\left\{u \in W^{1,1}\left(\Omega^{\prime}\right): \mathcal{F}\left(u ; \Omega^{\prime}\right)<\infty\right\} .
$$

It is clear that if we impose appropriate conditions at the boundary of a fixed open set $\Omega^{\prime} \Subset \Omega$, then from standard direct methods of the Calculus of Variations we derive the existence of minimizers in the Sobolev space

$$
W_{\text {loc }}^{1, \frac{p r}{r+1}}\left(\Omega^{\prime}\right),
$$

provided that $p r>r+1$. Furthermore, if $u \in W_{\text {loc }}^{1,1}(\Omega)$ is a quasi-minimizer of $\mathcal{F}$, it follows from (2.9) that

$$
u \in W_{\mathrm{loc}}^{1, \frac{p r}{r+1}}(\Omega) .
$$

For the sake of simplicity, we will explicitly assume this in our statements and we also introduce the following notation:

$$
\nu:=\frac{p r}{r+1},
$$

with the convention $\nu:=p$ if $r=+\infty$. It should be noticed that, from our assumptions on $r$ (that is, $r \geq 1$ and $r \geq 1 /(p-1)$ if $1<p<2)$, we easily infer that

$$
\nu=\frac{p r}{r+1} \geq 1 \text {. }
$$

Throughout the sequel, given any $\vartheta \in[1, \infty]$, we shall adopt the following notation: we indicate by $\vartheta^{\prime}$ the conjugate exponent of $\vartheta$, that is,

$$
\vartheta^{\prime}:= \begin{cases}\frac{\vartheta}{\vartheta-1}, & \text { if } \vartheta \in(1, \infty) ; \\ \infty, & \text { if } \vartheta=1 ; \\ 1, & \text { if } \vartheta=\infty .\end{cases}
$$

Moreover, we denote by $\vartheta^{*}$ the Sobolev exponent of $\vartheta$, that is,

$$
\vartheta^{*}:= \begin{cases}\frac{n \vartheta}{n-\vartheta}, & \text { if } \vartheta<n \\ \text { any number } t>\vartheta, & \text { if } \vartheta \geq n\end{cases}
$$

Our main result is contained in the following theorem.

Theorem 2.2. Let us consider the functional $\mathcal{F}$ in (2.1), and let us suppose that its integrand function $f$ satisfies (2.2), (2.3), (2.4), (2.5) and (2.6), with

$$
q \sigma^{\prime}<\nu^{*} \text {. }
$$

Let $u \in W_{\mathrm{loc}}^{1, \frac{p r}{r+1}}(\Omega)$ be a quasi-minimizer of $\mathcal{F}$.

Then $u$ is locally bounded in $\Omega$. Furthermore, it is possible to find a constant $c>0$, only depending on $n, p, q, r, \sigma, \tau$ and on $k$ and $Q$, with the following property: for every fixed $x_{0} \in \Omega$ and every $R_{0} \in(0,1]$ satisfying $B\left(x_{0}, R_{0}\right) \Subset \Omega$, one has

$$
\|u\|_{L^{\infty}\left(B_{R_{0} / 2}\left(x_{0}\right)\right)} \leq c\left(\frac{H}{R_{0}}\right)^{\frac{\nu^{*}}{\nu^{*}-q \sigma^{\prime}}}\left(1+\|u\|_{L^{\nu^{*}}\left(B_{R_{0}}\right)}\right)^{\frac{\nu^{*}(q-p+p \alpha)}{p\left(\nu^{*}-q \sigma^{\prime}\right)}}
$$


where

$$
H:=\left(1+\left\|\lambda^{-1}\right\|_{L^{r}\left(B\left(x_{0}, R_{0}\right)\right)}^{1 / p}\right)\left(1+\|a\|_{L^{\tau}\left(B\left(x_{0}, R_{0}\right)\right)}^{1 / p}\right)\left(1+\|\mu\|_{L^{\sigma}\left(B\left(x_{0}, R_{0}\right)\right)}^{1 / p}\right)
$$

and

$$
\alpha:=\min \left\{1-\frac{q \sigma^{\prime}}{\nu^{*}}, \sigma^{\prime}\left(1-\frac{1}{\tau}-\frac{p}{\nu^{*}}\right)\right\}
$$

We observe that, in the particular case when $q=p$, Theorem 2.2 reduces to Theorem 1.1 stated in the Introduction.

Example 2.3. Let us consider the functional

$$
\mathcal{F}(v)=\int_{\Omega} h(x)\left(|\nabla v(x)|^{p}+|u(x)|^{q}\right) \mathrm{d} x,
$$

where $1<p \leq q$ are real and $h$ is non-negative on $\Omega$. If we assume that

(i) $h^{-1} \in L_{\mathrm{loc}}^{r}(\Omega)$ and $h \in L_{\mathrm{loc}}^{\sigma}(\Omega)$, with

$$
\frac{1}{r}+\frac{1}{\sigma}<\frac{p}{n} \quad \text { and } \quad r \geq \max \left\{1, \frac{1}{p-1}\right\}
$$

(ii) $q<\nu^{*} / \sigma^{\prime}$ (remind that $\nu=p r /(r+1)$ and $\nu^{*}$ is Sobolev exponent of $\nu$ ), we infer from Theorem 2.2 that the quasi-minimizer of $\mathcal{F}$ are locally bounded.

Remark 2.4. Here, we list a few consequences of (2.6) and (2.12) which are of general interest.

(1) The first inequality in (2.6) implies

$$
p \sigma^{\prime}<\nu^{*} \quad \text { and } \quad 1-\frac{1}{\tau}-\frac{p}{\nu^{*}}>0 .
$$

(2) The assumption on $q$, see (2.12), together with the last inequality in (2.17), implies $\alpha>0$.

(3) If the function $a$ in the growth condition (2.3) is a locally bounded function, i.e., $\tau=+\infty$, we obtain

$$
\frac{1}{r}+\frac{1}{\sigma}<\frac{p}{n}
$$

It should be noticed that, in the particular case $p=2$, the above $(2.18)$ is precisely the assumption made by Trudinger in [23] (see indeed (1.7)).

(4) From the first inequality in (2.6) we get $\tau>n / p$. This bound from below for $\tau$ coincides with the condition in order to gain regularity for the minimizer in the standard case when $\lambda, \mu$ are constants.

(5) We notice that if $r=\sigma=+\infty$, i.e., $\lambda^{-1}, \mu \in L^{\infty}$, then (2.12) reduces to the well-known assumption $q<p^{*}$ (see for example Giusti [14]).

(6) If $r=\sigma=\tau=+\infty$ and $p=q$, then

$$
\frac{\nu^{*}(q-p+p \alpha)}{p\left(\nu^{*}-q \sigma^{\prime}\right)}=1
$$

and the estimate (2.13) becomes

$$
\|u\|_{L^{\infty}\left(B_{R_{0} / 2}\left(x_{0}\right)\right)} \leq c\left(\frac{H}{R_{0}}\right)^{\frac{p^{*}}{p^{*}-p}}\left(1+\|u\|_{L^{p^{*}}\left(B_{R_{0}}\right)}\right) .
$$




\section{CACCioppoli-type inequality}

Throughout the sequel, we shall make use of the following notation: given any function $u$ in $W_{\text {loc }}^{1,1}(\Omega)$, if $x_{0} \in \Omega$ and $R>0$ are such that $B\left(x_{0}, R\right) \Subset \Omega$ we set

$$
A_{\beta, R}=A_{\beta, R}(u):=\left\{x \in B\left(x_{0}, R\right): u(x)>\beta\right\}, \quad \beta \in \mathbb{R} .
$$

A fundamental tool for proving the local boundedness of the quasi-minimizers of $\mathcal{F}$ is the Caccioppoli-type inequality contained in the next Proposition 3.1. In the proof of this proposition we shall exploit a very classical lemma, see Lemma 3.2 below.

Proposition 3.1. Let $u \in W_{\mathrm{loc}}^{1, \frac{p r}{r+1}}(\Omega)$ be a quasi-minimizer of the functional $\mathcal{F}$ in (2.1); moreover, let $x_{0} \in \Omega$ and $R \in(0,1]$ be such that $B\left(x_{0}, R\right) \Subset \Omega$. Then, for every $\rho \in(0, R)$ and every $\beta \in \mathbb{R}$ with $\beta \geq 1$ we have

$$
\begin{gathered}
\int_{A_{\beta, \rho}} f(x, u, \nabla u) \mathrm{d} x \leq \Lambda\left\{\frac{1}{(R-\rho)^{p}} \int_{A_{\beta, R}} \mu(x)\left((u-\beta)^{p}+\beta^{q}\right) \mathrm{d} x\right. \\
\left.\quad+\|a\|_{L^{\tau}\left(B\left(x_{0}, R\right)\right)} \cdot\left|A_{\beta, R}\right|^{1-\frac{1}{\tau}}\right\},
\end{gathered}
$$

where $\Lambda>0$ is a constant only depending on $p$ and $Q$.

Proof. Let $\rho, \beta$ be as in the statement of the proposition, and let $s, t \in(0, R)$ be such that $\rho \leq s<t$. Moreover, let $\eta \in C_{c}^{\infty}\left(\mathbb{R}^{n}, \mathbb{R}\right)$ be a cut-off function satisfying the following properties:

(i) $0 \leq \eta \leq 1$ on $\mathbb{R}^{n}$;

(ii) $\operatorname{supp} \eta \Subset B\left(x_{0}, t\right)$ and $\eta \equiv 1$ on $B\left(x_{0}, s\right)$;

(iii) $|\nabla \eta(x)| \leq 2 /(t-s)$ for every $x \in \mathbb{R}^{n}$.

Setting $w:=(u-\beta)_{+}=\max \{u-\beta, 0\}$, we finally define

$$
\varphi: \Omega \rightarrow \mathbb{R}, \quad \varphi(x):=-\eta^{p}(x) w(x) .
$$

It is easy to check that

$$
\varphi \in W^{1,1}(\Omega) \text { and } A_{\beta, s} \subseteq \operatorname{supp} \varphi \subseteq A_{\beta, t} \Subset \Omega ;
$$

furthermore, we have

$$
\nabla w=\nabla u \text { on } \operatorname{supp} \varphi .
$$

Since $u$ is a quasi-minimizer of $\mathcal{F}$ with constant $Q \geq 1$, and since $f \geq 0$, one has

$$
\begin{aligned}
& \int_{A_{\beta, s}} f(x, u, \nabla u) \mathrm{d} x \leq \int_{\operatorname{supp} \varphi} f(x, u, \nabla u) \mathrm{d} x \\
& \leq Q \int_{\operatorname{supp} \varphi} f(x, u+\varphi, \nabla(u+\varphi)) \mathrm{d} x \\
& \stackrel{(3.4)}{=} Q \int_{\operatorname{supp} \varphi} f\left(x,\left(1-\eta^{p}\right) u+\eta^{p} \beta,\left(1-\eta^{p}\right) \nabla u+\eta^{p}\left(p w \eta^{-1} \nabla \eta\right)\right) \mathrm{d} x \\
& \quad \stackrel{(2.2)}{\leq} Q \int_{\operatorname{supp} \varphi}\left\{\left(1-\eta^{p}\right) f(x, u, \nabla u)+\eta^{p} f\left(x, \beta, p w \eta^{-1} \nabla \eta\right)\right\} \mathrm{d} x .
\end{aligned}
$$

We now introduce the notation

$$
\begin{aligned}
& \mathrm{I}_{1}:=\int_{\operatorname{supp} \varphi}\left(1-\eta^{p}\right) f(x, u, \nabla u) \mathrm{d} x \\
& \quad \mathrm{I}_{2}:=\int_{\operatorname{supp} \varphi} \eta^{p} f\left(x, \beta, p w \eta^{-1} \nabla \eta\right) \mathrm{d} x,
\end{aligned}
$$

and we proceed by giving an estimate of them. 
As regards $\mathrm{I}_{1}$, since $\eta \equiv 1$ on $B\left(x_{0}, s\right)$ and since $0 \leq \eta \leq 1$ on the whole of $\mathbb{R}^{n}$, we immediately obtain

$$
\begin{aligned}
\mathrm{I}_{1} & \stackrel{(3.3)}{\leq} \int_{A_{\beta, t}}\left(1-\eta^{p}\right) f(x, u, \nabla u) \mathrm{d} x \\
& =\int_{A_{\beta, t} \backslash A_{\beta, s}}\left(1-\eta^{p}\right) f(x, u, \nabla u) \mathrm{d} x \\
& \leq \int_{A_{\beta, t} \backslash A_{\beta, s}} f(x, u, \nabla u) \mathrm{d} x .
\end{aligned}
$$

We then turn our attention to $\mathrm{I}_{2}$. On account of $(2.3)$, one has

$$
\begin{aligned}
\mathrm{I}_{2} & \left.\leq \int_{\operatorname{supp} \varphi} \eta^{p}\left\{\mu(x)\left(p w \eta^{-1}\right)^{p}|\nabla \eta|^{p}+\beta^{q}\right)+a(x)\right\} \mathrm{d} x \\
& \leq \int_{\operatorname{supp} \varphi} \mu(x)\left((p w)^{p}|\nabla \eta|^{p}+\beta^{q}\right) \mathrm{d} x+\int_{\operatorname{supp} \varphi} a(x) \mathrm{d} x=:(\star) ;
\end{aligned}
$$

moreover, by using the estimate for $|\nabla \eta|$ in (iii) and since $0<t-s \leq R \leq 1$ and $p>1$, we get

$$
\begin{aligned}
(\star & \leq \int_{\operatorname{supp} \varphi} \mu(x)\left(\frac{(2 p)^{p}}{(t-s)^{p}} w^{p}+\beta^{q}\right) \mathrm{d} x+\int_{\operatorname{supp} \varphi} a(x) \mathrm{d} x \\
& \leq \frac{(2 p)^{p}}{(t-s)^{p}} \int_{\operatorname{supp} \varphi} \mu(x)\left(w^{p}+\beta^{q}\right) \mathrm{d} x+\int_{\operatorname{supp} \varphi} a(x) \mathrm{d} x=:(2 \star) .
\end{aligned}
$$

By applying Hölder's inequality, using (3.3), (2.5) and noting that $w=(u-\beta)_{+}$, we finally get

$$
\begin{aligned}
(2 \star) & \leq \frac{(2 p)^{p}}{(t-s)^{p}} \int_{A_{\beta, t}} \mu(x)\left(w^{p}+\beta^{q}\right) \mathrm{d} x+\int_{A_{\beta, t}} a(x) \mathrm{d} x \\
& \leq \frac{(2 p)^{p}}{(t-s)^{p}} \int_{A_{\beta, t}} \mu(x)\left(w^{p}+\beta^{q}\right) \mathrm{d} x+\|a\|_{L^{\tau}\left(B\left(x_{0}, R\right)\right)}\left|A_{\beta, R}\right|^{1-\frac{1}{\tau}} \\
& =\frac{(2 p)^{p}}{(t-s)^{p}} \int_{A_{\beta, R}} \mu(x)\left((u-\beta)^{p}+\beta^{q}\right) \mathrm{d} x+\|a\|_{L^{\tau}\left(B\left(x_{0}, R\right)\right)}\left|A_{\beta, R}\right|^{1-\frac{1}{\tau}} .
\end{aligned}
$$

Gathering together (3.5) and (3.6), we then obtain

$$
\begin{gathered}
\int_{A_{\beta, s}} f(x, u, \nabla u) \mathrm{d} x \leq Q \int_{A_{\beta, t} \backslash A_{\beta, s}} f(x, u, \nabla u) \mathrm{d} x \\
+Q\left(\frac{(2 p)^{p}}{(t-s)^{p}} \int_{A_{\beta, R}} \mu(x)\left(|u-\beta|^{p}+\beta^{q}\right) \mathrm{d} x\right. \\
\left.+\|a\|_{L^{\tau}\left(B\left(x_{0}, R\right)\right)}\left|A_{\beta, R}\right|^{1-\frac{1}{\tau}}\right) .
\end{gathered}
$$

By the hole-filling method, this last estimate clearly implies that

$$
\begin{gathered}
\int_{A_{\beta, s}} f(x, u, \nabla u) \mathrm{d} x \leq \frac{Q}{Q+1} \int_{A_{\beta, t}} f(x, u, \nabla u) \mathrm{d} x \\
+\left(\frac{(2 p)^{p}}{(t-s)^{p}} \int_{A_{\beta, R}} \mu(x)\left((u-\beta)^{p}+\beta^{q}\right) \mathrm{d} x\right. \\
\left.+\|a\|_{L^{\tau}\left(B\left(x_{0}, R\right)\right)}\left|A_{\beta, R}\right|^{1-\frac{1}{\tau}}\right) .
\end{gathered}
$$


We are now entitled to apply Lemma 3.2 below, with

$$
\omega:[\rho, R] \rightarrow \mathbb{R}, \quad \omega(t):=\int_{A_{\beta, t}} f(x, u, \nabla u) \mathrm{d} x,
$$

and the constants $\vartheta, \kappa, A$ and $B$ given, respectively, by

$$
\begin{gathered}
\vartheta=\frac{Q}{Q+1}, \quad \kappa=p, \quad A:=(2 p)^{p} \int_{A_{\beta, R}} \mu(x)\left((u-\beta)^{p}+\beta^{q}\right) \mathrm{d} x \\
\text { and } \quad B:=\|a\|_{L^{\tau}\left(B\left(x_{0}, R\right)\right)}\left|A_{\beta, R}\right|^{1-\frac{1}{\tau}} .
\end{gathered}
$$

Thus, we find a constant $\Lambda>0$, only depending on $\kappa=p$ and on $Q$, such that

$$
\begin{gathered}
\omega(\rho)=\int_{A_{\beta, \rho}} f(x, u, \nabla u) \mathrm{d} x \\
\leq \Lambda\left\{\frac{1}{(R-\rho)^{p}} \int_{A_{\beta, R}} \mu(x)\left((u-\beta)^{p}+\beta^{q}\right) \mathrm{d} x\right. \\
\left.\quad+\|a\|_{L^{\tau}\left(B\left(x_{0}, R\right)\right)}\left|A_{\beta, R}\right|^{1-\frac{1}{\tau}}\right\} .
\end{gathered}
$$

The proof is complete.

We now state the classical lemma used in the proof above. We refer, e.g., to [14, Lemma 6.1] for a demonstration of this result.

Lemma 3.2. Let $[a, b] \subseteq \mathbb{R}$ and let $\omega:[a, b] \rightarrow \mathbb{R}$ be non-negative and bounded. We assume that there exist constants $\kappa, A, B>0$ and $\vartheta \in(0,1)$ such that

$$
\omega(s) \leq \vartheta \omega(t)+\frac{A}{(t-s)^{\kappa}}+B, \quad \text { for every } a \leq s \leq t \leq b .
$$

It is then possible to find a constant $C=C(\vartheta, \kappa)>0$ such that

$$
\omega(\rho) \leq C\left\{\frac{A}{(R-\rho)^{\kappa}}+B\right\}, \quad \text { for every } a \leq \rho \leq R \leq b .
$$

\section{Proof of Theorem 2.2}

Now we proceed towards the proof of our Theorem 2.2 by setting up the celebrated De Giorgi's iterative method. In what follows, we tacitly understand that all the assumptions and the notation introduced in the previous sections do apply.

Let $u \in W_{\text {loc }}^{1, \frac{p r}{r+1}}(\Omega)$ be a quasi-minimizer of $\mathcal{F}$ in (2.1). Taking into account Remark 2.4 , we fix a point $x_{0} \in \Omega$ and a real $R_{0} \in(0,1]$ in such a way that

$$
B\left(x_{0}, R_{0}\right) \Subset \Omega \quad \text { and } \quad \int_{B\left(x_{0}, R_{0}\right)}|u|^{p \sigma^{\prime}} \mathrm{d} x \leq 1 .
$$

For every fixed $R \in\left(0, R_{0}\right]$, we then define the following (decreasing) sequences:

$$
\rho_{h}:=\frac{R}{2}\left(1+\frac{1}{2^{h}}\right) \quad \text { and } \quad \bar{\rho}_{h}:=\frac{\rho_{h+1}+\rho_{h}}{2}, \quad h \in \mathbb{N} \cup\{0\} .
$$

Moreover, given any real number $\beta \geq 1$, we consider the (increasing) sequence

$$
\beta_{h}:=\beta\left(1-\frac{1}{2^{h+1}}\right), \quad h \in \mathbb{N} \cup\{0\} .
$$

Finally, we define a sequence $\left(J_{h}\right)_{h \geq 0}$ of non-negative numbers as follows:

$$
J_{h}:=\int_{A_{\beta_{h}, \rho_{h}}}\left(u-\beta_{h}\right)^{p \sigma^{\prime}} \mathrm{d} x .
$$


We explicitly observe that, since $\left(\rho_{h}\right)_{h}$ is decreasing and $\left(\beta_{h}\right)_{h}$ is increasing, the sequence $\left(J_{h}\right)_{h}$ is decreasing: in fact, we have

$$
\begin{aligned}
J_{h+1} & =\int_{A_{\beta_{h+1}, \rho_{h+1}}}\left(u-\beta_{h+1}\right)^{p \sigma^{\prime}} \mathrm{d} x \leq \int_{A_{\beta_{h+1}, \rho_{h}}}\left(u-\beta_{h+1}\right)^{p \sigma^{\prime}} \mathrm{d} x \\
& \leq \int_{A_{\beta_{h+1}, \rho_{h}}}\left(u-\beta_{h}\right)^{p \sigma^{\prime}} \mathrm{d} x \leq \int_{A_{\beta_{h}, \rho_{h}}}\left(u-\beta_{h}\right)^{p \sigma^{\prime}} \mathrm{d} x=J_{h} .
\end{aligned}
$$

Moreover, on account of (4.1) (and since $\rho_{h} \leq R \leq R_{0}$ ), we have

$$
\begin{aligned}
J_{h} & =\int_{A_{\beta_{h}, \rho_{h}}}\left(u-\beta_{h}\right)^{p \sigma^{\prime}} \mathrm{d} x \leq \int_{A_{\beta_{h}, \rho_{h}}}|u|^{p \sigma^{\prime}} \mathrm{d} x \\
& \leq \int_{B\left(x_{0}, R_{0}\right)}|u|^{p \sigma^{\prime}} \mathrm{d} x \leq 1 .
\end{aligned}
$$

Finally, by taking into account the definitions of $J_{h}, \beta_{h}$ and $\rho_{h}$, we have

$$
\begin{aligned}
J_{h} & =\int_{A_{\beta_{h}, \rho_{h}}}\left(u-\beta_{h}\right)^{p \sigma^{\prime}} \mathrm{d} x \geq \int_{A_{\beta_{h+1}, \rho_{h}}}\left(u-\beta_{h}\right)^{p \sigma^{\prime}} \mathrm{d} x \\
& \geq\left(\beta_{h+1}-\beta_{h}\right)^{p \sigma^{\prime}}\left|A_{\beta_{h+1}, \rho_{h}}\right|=\left(\frac{\beta}{2^{h+2}}\right)^{p \sigma^{\prime}}\left|A_{\beta_{h+1}, \rho_{h}}\right|,
\end{aligned}
$$

The following proposition is the key tool for the proof of Theorem 2.2 .

Proposition 4.1. Let the above assumptions and notation do apply. Then, there exists a constant $C>0$ such that, for every $R \in\left(0, R_{0}\right]$ and every $\beta \geq 1$, one has

$$
J_{h+1} \leq C\left(1+\|u\|_{L^{\nu *}\left(B\left(x_{0}, R\right)\right)}^{(q-p) \sigma^{\prime}}\right) \cdot \frac{8^{p \sigma^{\prime 2} h}}{R^{p \sigma^{\prime}} \beta^{p \sigma^{\prime}\left(1-\frac{q \sigma^{\prime}}{\nu^{*}}\right)}} J_{h}^{1+\alpha} \quad \forall h \in \mathbb{N} \cup\{0\},
$$

where $\nu$ is as in (2.10) and $\alpha$ is as in (2.15). Furthermore, the dependence of $C$ with respect to $\lambda, \mu$, a can be explicated as follows:

$$
C=c\left\{\left\|\lambda^{-1}\right\|_{L^{r}\left(B\left(x_{0}, R_{0}\right)\right)} \cdot\left(1+\|a\|_{L^{\tau}\left(B\left(x_{0}, R_{0}\right)\right)}\right) \cdot\left(1+\|\mu\|_{L^{\sigma}\left(B\left(x_{0}, R_{0}\right)\right)}\right)\right\}^{\sigma^{\prime}},
$$

where $c>0$ is constant depending on $n, p, q, r, \sigma, \tau$, on $k$ in (2.4) and on $Q$.

Before proving Proposition 4.1 we need a preliminary lemma (for a proof see, e.g., [7], Lemma 3.2).

Lemma 4.2. Let $p>1$ be fixed and let $r \geq \max \left\{1, \frac{1}{p-1}\right\}$. As usual, we set

$$
\nu:=\frac{p r}{r+1} .
$$

Then, there exists a constant $c_{S}>0$ such that

$$
\left(\int_{B}|v|^{\nu^{*}} \mathrm{~d} x\right)^{p / \nu^{*}} \leq c_{S}\left\|\lambda^{-1}\right\|_{L^{r}(B)} \int_{B} \lambda(x)|\nabla v|^{p} \mathrm{~d} x,
$$

for every ball $B \Subset \Omega$ and every function $v \in W_{0}^{1, \frac{p r}{r+1}}(B)$.

Now, we can provide the proof of Proposition 4.1.

Proof of Proposition 4.1. Let $\left(\eta_{h}\right)_{h \geq 0}$ be a sequence in $C_{c}^{\infty}\left(\mathbb{R}^{n}\right)$ such that

(i) $0 \leq \eta_{h} \leq 1$ on $\mathbb{R}^{n}$;

(ii) $\operatorname{supp} \eta_{h} \subseteq B\left(x_{0}, \bar{\rho}_{h}\right)$ and $\eta_{h} \equiv 1$ on $B\left(x_{0}, \rho_{h+1}\right)$;

(iii) $\left|\nabla \eta_{h}\right| \leq \frac{2^{h+4}}{R}$. 
We remind here that

$$
\nu:=\frac{p r}{r+1}
$$

Since $p \sigma^{\prime}<\nu^{*}$ (see Remark 2.4-(1)), and reminding that

$$
\eta_{h} \equiv 1 \text { on } A_{\beta_{h+1}, \rho_{h+1}} \quad \text { and } \quad \operatorname{supp} \eta_{h} \subseteq B\left(x_{0}, \bar{\rho}_{h}\right)
$$

we have

$$
\begin{aligned}
J_{h+1} & =\int_{A_{\beta_{h+1}, \rho_{h+1}}}\left(u-\beta_{h+1}\right)^{p \sigma^{\prime}} \mathrm{d} x \\
& \left.\leq\left|A_{\beta_{h+1}, \rho_{h+1}}\right|^{1-\frac{p \sigma^{\prime}}{\nu^{*}}}\left(\int_{A_{\beta_{h+1}, \rho_{h+1}}}\left(u-\beta_{h+1}\right)^{\nu^{*}} \mathrm{~d} x\right)^{\frac{p \sigma^{\prime}}{\nu^{*}}} \eta_{h}\left(u-\beta_{h+1}\right)^{\nu^{*}} \mathrm{~d} x\right)^{\frac{p \sigma^{\prime}}{\nu^{*}}} \\
& =\left|A_{\beta_{h+1}, \rho_{h+1}}\right|^{1-\frac{p \sigma^{\prime}}{\nu^{*}}}\left(\int_{A_{\beta_{h+1}, \rho_{h+1}}} \eta_{h}\left(u-\beta_{h+1}\right)_{+}^{\nu^{*}} \mathrm{~d} x\right)^{\frac{p \sigma^{\prime}}{\nu^{*}}}=:(\star) . \\
& \leq\left|A_{\beta_{h+1}, \rho_{h+1}}\right|^{1-\frac{p \sigma^{\prime}}{\nu^{*}}}\left(\int_{B\left(x_{0}, \bar{\rho}_{h}\right)}\right.
\end{aligned}
$$

To proceed further we observe that, by the fact that $\eta_{h} \in C_{c}^{\infty}\left(B\left(x_{0}, \bar{\rho}_{h}\right)\right)$ (see (ii)), we certainly have $v:=\eta_{h}\left(u-\beta_{h+1}\right)_{+} \in W_{0}^{1, \nu}\left(B\left(x_{0}, \bar{\rho}_{h}\right)\right)$. We are then entitled to apply Lemma 4.2 , with $\Omega^{\prime}:=B\left(x_{0}, \bar{\rho}_{h}\right) \Subset B\left(x_{0}, R\right)$. If we denote

$$
K:=\left\|\lambda^{-1}\right\|_{L^{r}\left(B\left(x_{0}, R_{0}\right)\right)}^{\sigma^{\prime}},
$$

we have (here $c_{S}$ is the embedding constant in Lemma 4.2)

$$
\begin{aligned}
(\star) \leq & c_{S}^{\sigma^{\prime}} K\left|A_{\beta_{h+1}, \rho_{h+1}}\right|^{1-\frac{p \sigma^{\prime}}{\nu^{*}}}\left(\int_{B\left(x_{0}, \bar{\rho}_{h}\right)} \lambda(x)\left|\nabla\left(\eta_{h}\left(u-\beta_{h+1}\right)_{+}\right)\right|^{p} \mathrm{~d} x\right)^{\sigma^{\prime}} \\
\leq & c_{S}^{\sigma^{\prime}} K\left|A_{\beta_{h+1}, \rho_{h+1}}\right|^{1-\frac{p \sigma^{\prime}}{\nu^{*}}} \times \\
& \times\left(\int_{B\left(x_{0}, \bar{\rho}_{h}\right)} \lambda(x)\left\{\left|\nabla \eta_{h}\right|\left(u-\beta_{h+1}\right)_{+}+\eta_{h}|\nabla u| \chi_{A_{\beta_{h+1}, \bar{\rho}_{h}}}\right\}^{p} \mathrm{~d} x\right)^{\sigma^{\prime}}=:(2 \star) .
\end{aligned}
$$

By the estimate for $\left|\nabla \eta_{h}\right|$ in (iii), using $\eta_{h} \leq 1$ and inequality (2.3) we get (here and throughout, we indicate by $c_{1}, c_{2}, \ldots$ different positive constants only depending on the Sobolev constant $c_{S}$, on $n, p, q, r, \sigma, \tau$, on $k$ in (2.4) and on $Q$ )

$$
\begin{aligned}
& \int_{B\left(x_{0}, \bar{\rho}_{h}\right)} \lambda(x)\left\{\left|\nabla \eta_{h}\right|\left(u-\beta_{h+1}\right)_{+}+\eta_{h}|\nabla u| \chi_{A_{\beta_{h+1}, \bar{\rho}_{h}}}\right\}^{p} \mathrm{~d} x \\
& \leq c_{1}\left\{\left(\frac{2^{h+4}}{R}\right)^{p} \int_{A_{\beta_{h+1}, \bar{\rho} h}} \lambda(x)\left(u-\beta_{h+1}\right)^{p} \mathrm{~d} x+\int_{A_{\beta_{h+1}, \bar{\rho} h}} \lambda(x)|\nabla u|^{p} \mathrm{~d} x\right\} \\
& \leq c_{1}\left\{\left(\frac{2^{h+4}}{R}\right)^{p} \int_{A_{\beta_{h+1}, \bar{\rho} h}} \lambda(x)\left(u-\beta_{h+1}\right)^{p} \mathrm{~d} x+\int_{A_{\beta_{h+1}, \bar{\rho}_{h}}} f(x, u, \nabla u) \mathrm{d} x\right\} .
\end{aligned}
$$


Collecting the above inequalities and by exploiting the Caccioppoli-type inequality (3.2) established in Section 3, we then obtain

$$
\begin{aligned}
& (2 \star) \stackrel{(2.3)}{\leq} c_{1} K\left|A_{\beta_{h+1}, \rho_{h+1}}\right|^{1-\frac{p \sigma^{\prime}}{\nu^{*}}}\left[\left(\frac{2^{h+4}}{R}\right)^{p} \int_{A_{\beta_{h+1}, \bar{\rho} h}} \lambda(x)\left(u-\beta_{h+1}\right)^{p} \mathrm{~d} x\right. \\
& \left.+\int_{A_{\beta_{h+1}, \bar{\rho}_{h}}} f(x, u, \nabla u) \mathrm{d} x\right]^{\sigma^{\prime}}
\end{aligned}
$$

(by $(3.2)$, with $\rho=\bar{\rho}_{h}$ and $R=\rho_{h}$ )

$$
\begin{gathered}
\leq c_{1} K\left|A_{\beta_{h+1}, \rho_{h+1}}\right|^{1-\frac{p \sigma^{\prime}}{\nu^{*}}}\left\{\left(\frac{2^{h+4}}{R}\right)^{p} \int_{A_{\beta_{h+1}, \bar{\rho}_{h}}} \lambda(x)\left(u-\beta_{h+1}\right)^{p} \mathrm{~d} x\right. \\
+\Lambda\left[\left(\frac{2^{h+3}}{R}\right)^{p} \int_{A_{\beta_{h+1}, \rho_{h}}} \mu(x)\left(\left(u-\beta_{h+1}\right)^{p}+\beta_{h+1}^{q}\right) \mathrm{d} x\right. \\
\left.\left.+\|a\|_{L^{\tau}\left(B\left(x_{0}, R\right)\right)}\left|A_{\beta_{h+1}, \rho_{h}}\right|^{1-\frac{1}{\tau}}\right]\right\}^{\sigma^{\prime}}=:(3 \star) .
\end{gathered}
$$

Let us now recall that $(2.4)$ holds and $R \leq 1$. Therefore, setting

$$
K_{1}:=K \cdot\left(1+\|a\|_{L^{\tau}\left(B\left(x_{0}, R_{0}\right)\right)}\right)^{\sigma^{\prime}} \quad \text { and } \quad K_{2}:=K_{1} \cdot\left(1+\|\mu\|_{L^{\sigma}\left(B\left(x_{0}, R_{0}\right)\right)}\right)^{\sigma^{\prime}},
$$

by Hölder's inequality we obtain

$$
\begin{aligned}
(3 \star) \leq & c_{2} K_{1}\left|A_{\beta_{h+1}, \rho_{h+1}}\right|^{1-\frac{p \sigma^{\prime}}{\nu^{*}}}\left(\frac{2^{h}}{R}\right)^{p \sigma^{\prime}} \times \\
\times & \left.\times \int_{A_{\beta_{h+1}, \rho_{h}}} \mu(x)\left(\left(u-\beta_{h+1}\right)^{p}+\beta_{h+1}^{q}\right) \mathrm{d} x+\left|A_{\beta_{h+1}, \rho_{h}}\right|^{1-\frac{1}{\tau}}\right]^{\sigma^{\prime}} \\
\leq & c_{2} K_{1}\left|A_{\beta_{h+1}, \rho_{h+1}}\right|^{1-\frac{p \sigma^{\prime}}{\nu^{*}}}\left(\frac{2^{h}}{R}\right)^{p \sigma^{\prime}} \times \\
& \times \quad\left[\|\mu\|_{L^{\sigma}\left(B\left(x_{0}, R\right)\right)}\left(\int_{A_{\beta_{h+1}, \rho_{h}}}\left(\left(u-\beta_{h+1}\right)^{p}+\beta_{h+1}^{q}\right)^{\sigma^{\prime}} \mathrm{d} x\right)^{\frac{1}{\sigma^{\prime}}}\right. \\
& \left.+\left|A_{\beta_{h+1}, \rho_{h}}\right|^{1-\frac{1}{\tau}}\right]^{\sigma^{\prime}} \\
\leq & c_{3} K_{2}\left|A_{\beta_{h+1}, \rho_{h+1}}\right|^{1-\frac{p \sigma^{\prime}}{\nu^{*}}}\left(\frac{2^{h}}{R}\right)^{p \sigma^{\prime}} \times \\
& \left\{\int_{A_{\beta_{h+1}, \rho_{h}}}\left(\left(u-\beta_{h+1}\right)^{p}+\beta_{h+1}^{q}\right)^{\sigma^{\prime}} \mathrm{d} x+\left|A_{\beta_{h+1}, \rho_{h}}\right|^{\sigma^{\prime}\left(1-\frac{1}{\tau}\right)}\right\}=:(4 \star)
\end{aligned}
$$

It holds that

$$
\begin{aligned}
& \int_{A_{\beta_{h+1}, \rho_{h}}}\left(\left(u-\beta_{h+1}\right)^{p}+\beta_{h+1}^{q}\right)^{\sigma^{\prime}} \mathrm{d} x \leq c_{\sigma^{\prime}}\left\{\int_{A_{\beta_{h+1}, \rho_{h}}}\left(u-\beta_{h+1}\right)^{p \sigma^{\prime}} \mathrm{d} x\right. \\
& \left.+\int_{A_{\beta_{h+1}, \rho_{h}}} \beta_{h+1}^{q \sigma^{\prime}} \mathrm{d} x\right\} .
\end{aligned}
$$

Let us now estimate the last integral. Since

$$
\beta_{h+1}^{\nu^{*}}\left|A_{\beta_{h+1}, \rho_{h}}\right| \leq \int_{A_{\beta_{h+1}, \rho_{h}}}|u|^{\nu^{*}} d x \leq\|u\|_{L^{\nu^{*}}\left(B\left(x_{0}, R\right)\right)}^{\nu^{*}},
$$


we have (remind that $\beta_{h} \leq \beta$ for any $h \geq 0$ )

$$
\begin{aligned}
\int_{A_{\beta_{h+1}, \rho_{h}}} \beta_{h+1}^{q \sigma^{\prime}} \mathrm{d} x & =\left(\beta_{h+1}^{\nu^{*}}\left|A_{\beta_{h+1}, \rho_{h}}\right|\right)^{\frac{(q-p) \sigma^{\prime}}{\nu^{*}}} \beta_{h+1}^{p \sigma^{\prime}}\left|A_{\beta_{h+1}, \rho_{h}}\right|^{1-\frac{(q-p) \sigma^{\prime}}{\nu^{*}}} \\
& \leq\|u\|_{L^{\nu^{*}}\left(B\left(x_{0}, R\right)\right)}^{(q-p) \sigma^{\prime}} \beta^{p \sigma^{\prime}}\left|A_{\beta_{h+1}, \rho_{h}}\right|^{1-\frac{(q-p) \sigma^{\prime}}{\nu^{*}}} .
\end{aligned}
$$

Therefore

$$
\begin{aligned}
& \int_{A_{\beta_{h+1}, \rho_{h}}}\left(\left(u-\beta_{h+1}\right)^{p}+\beta_{h+1}^{q}\right)^{\sigma^{\prime}} \mathrm{d} x \\
& \leq c_{\sigma^{\prime}} \int_{A_{\beta_{h+1}, \rho_{h}}}\left(u-\beta_{h+1}\right)^{p{\sigma^{\prime}}^{\prime}} \mathrm{d} x+c_{\sigma^{\prime}}\|u\|_{L^{\nu^{*}\left(B\left(x_{0}, R\right)\right)}}^{(q-p) \sigma^{\prime}} \beta^{p \sigma^{\prime}}\left|A_{\beta_{h+1}, \rho_{h}}\right|^{1-\frac{(q-p) \sigma^{\prime}}{\nu^{*}}} \\
& \leq c_{\sigma^{\prime}} J_{h}+c_{\sigma^{\prime}}\|u\|_{L^{\nu^{*}}\left(B\left(x_{0}, R\right)\right)}^{(q-p) \sigma^{\prime}} \beta^{p \sigma^{\prime}}\left|A_{\beta_{h+1}, \rho_{h}}\right|^{1-\frac{(q-p) \sigma^{\prime}}{\nu^{*}}}
\end{aligned}
$$

where in the last inequality we used (4.5). Now, owing to (4.7) we get

$$
\left|A_{\beta_{h+1}, \rho_{h+1}}\right| \leq\left|A_{\beta_{h+1}, \rho_{h}}\right| \leq\left(\frac{2^{h+2}}{\beta}\right)^{p \sigma^{\prime}} J_{h}=4^{p \sigma^{\prime}}\left(\frac{2^{h}}{\beta}\right)^{p \sigma^{\prime}} J_{h}
$$

this, together with (4.11) and the assumptions $q \geq p$ and $J_{h} \leq 1$, implies

$$
\begin{aligned}
& \int_{A_{\beta_{h+1}, \rho_{h}}}\left(\left(u-\beta_{h+1}\right)^{p}+\beta_{h+1}^{q}\right)^{\sigma^{\prime}} \mathrm{d} x+\left|A_{\beta_{h+1}, \rho_{h}}\right|^{\sigma^{\prime}\left(1-\frac{1}{\tau}\right)} \\
& \leq c_{4}\left(1+\|u\|_{L^{\nu^{*}}\left(B\left(x_{0}, R\right)\right)}^{(q-p) \sigma^{\prime}}\right)\left\{J_{h}+2^{h p \sigma^{\prime}\left(1-\frac{(q-p) \sigma^{\prime}}{\nu^{*}}\right)} \beta^{p \sigma^{\prime}-p \sigma^{\prime}\left(1-\frac{(q-p) \sigma^{\prime}}{\nu^{*}}\right)} J_{h}^{1-\frac{(q-p) \sigma^{\prime}}{\nu^{*}}}\right. \\
& \left.+\left(\frac{2^{h}}{\beta}\right)^{p \sigma^{\prime 2}\left(1-\frac{1}{\tau}\right)} J_{h}^{\sigma^{\prime}\left(1-\frac{1}{\tau}\right)}\right\} \\
& \leq c_{4}\left(1+\|u\|_{L^{\nu^{*}}\left(B\left(x_{0}, R\right)\right)}^{(q-p) \sigma^{\prime}}\right) 2^{h p \sigma^{\prime 2}} \beta^{\frac{p(q-p) \sigma^{\prime 2}}{\nu^{*}}} J_{h}^{\theta},
\end{aligned}
$$

where we have introduced the constant

$$
\theta:=\min \left\{1-\frac{(q-p) \sigma^{\prime}}{\nu^{*}}, \sigma^{\prime}\left(1-\frac{1}{\tau}\right)\right\} .
$$

Thus, we get

$$
\begin{aligned}
(4 \star) & \leq c_{5} K_{2}\left(1+\|u\|_{L^{\nu^{*}}\left(B\left(x_{0}, R\right)\right)}^{(q-p) \sigma^{\prime}}\right) \cdot \frac{2^{2 p \sigma^{\prime 2} h}}{R^{p \sigma^{\prime}}}\left|A_{\beta_{h+1}, \rho_{h+1}}\right|^{1-\frac{p \sigma^{\prime}}{\nu^{*}}} \beta^{\frac{p(q-p) \sigma^{\prime 2}}{\nu^{*}}} J_{h}^{\theta} \\
& \stackrel{(4.12)}{\leq} c_{6} K_{2}\left(1+\|u\|_{L^{\nu^{*}}\left(B\left(x_{0}, R\right)\right)}^{(q-p) \sigma^{\prime}}\right) \cdot \frac{2^{3 p \sigma^{\prime 2} h}}{R^{p \sigma^{\prime}}} \frac{\beta^{\frac{p(q-p) \sigma^{\prime 2}}{\nu^{*}}}}{\beta^{p \sigma^{\prime}\left(1-\frac{p \sigma^{\prime}}{\nu^{*}}\right)}} J_{h}^{1+\theta-\frac{p \sigma^{\prime}}{\nu^{*}}} \\
& \leq c_{6} K_{2}\left(1+\|u\|_{L^{\nu^{*}}\left(B\left(x_{0}, R\right)\right)}^{(q-p) \sigma^{\prime}}\right) \cdot \frac{8^{p \sigma^{\prime 2} h}}{R^{p \sigma^{\prime}}} \frac{1}{\beta^{p \sigma^{\prime}\left(1-\frac{q \sigma^{\prime}}{\nu^{*}}\right)}} J_{h}^{1+\theta-\frac{p \sigma^{\prime}}{\nu^{*}}} .
\end{aligned}
$$

Since, by the very definition of $\theta$, we have

$$
\alpha=\theta-\frac{p \sigma^{\prime}}{\nu^{*}}=\min \left\{1-\frac{(q-p) \sigma^{\prime}}{\nu^{*}}-\frac{p \sigma^{\prime}}{\nu^{*}}, \sigma^{\prime}\left(1-\frac{1}{\tau}\right)-\frac{p \sigma^{\prime}}{\nu^{*}}\right\},
$$

by collecting the previous chain of inequalities we get (4.8) with

$$
\begin{aligned}
C & =c_{6} K_{2} \\
& =c_{6}\left\{\left\|\lambda^{-1}\right\|_{L^{r}\left(B\left(x_{0}, R_{0}\right)\right)} \cdot\left(1+\|a\|_{L^{\tau}\left(B\left(x_{0}, R_{0}\right)\right)}\right) \cdot\left(1+\|\mu\|_{L^{\sigma}\left(B\left(x_{0}, R_{0}\right)\right)}\right)\right\}^{\sigma^{\prime}} .
\end{aligned}
$$


This ends the demonstration.

With Proposition 4.1 at hand, we are ready to provide the proof of our main result, namely Theorem 2.2. Before doing this, we remind the following very classical lemma of Real Analysis (see, e.g., [14, Lemma 7.1]).

Lemma 4.3. Let $\left(z_{h}\right)_{h \geq 0}$ be a sequence of positive real numbers satisfying the following recursive relation

$$
z_{h+1} \leq L \zeta^{h} z_{h}^{1+\gamma} \quad(h \in \mathbb{N} \cup\{0\}),
$$

where $L, \gamma>0$ and $\zeta>1$. If $z_{0} \leq L^{-\frac{1}{\gamma}} \zeta^{-\frac{1}{\gamma^{2}}}$, then

$$
z_{h} \leq \zeta^{-\frac{h}{\gamma}} z_{0} \quad \text { for every } h \geq 0 .
$$

In particular, $z_{h} \rightarrow 0$ as $h \rightarrow \infty$.

We now prove Theorem 2.2.

Proof of Theorem 2.2. Let $u \in W_{\text {loc }}^{1, \frac{p r}{r+1}}(\Omega)$ be a quasi-minimizer of the functional $\mathcal{F}$ in $(2.1)$, let $x_{0} \in \Omega$ be fixed and let $R_{0} \in(0,1]$ be such that $B\left(x_{0}, R_{0}\right) \Subset \Omega$. Moreover, let $\beta \geq 1$ (to be chosen later on) and let $\left(J_{h}\right)_{h \geq 0}$ be the sequence defined in (4.4). Owing to Proposition 4.1 (with $R=R_{0}$ ), we have the estimate

$$
J_{h+1} \leq L\left(8^{p \sigma^{\prime 2}}\right)^{h} J_{h}^{1+\alpha} \quad(h \in \mathbb{N} \cup\{0\}),
$$

where $\alpha$ is as in (2.15), that is,

$$
\alpha:=\min \left\{1-\frac{q \sigma^{\prime}}{\nu^{*}}, \sigma^{\prime}\left(1-\frac{1}{\tau}-\frac{p}{\nu^{*}}\right)\right\}
$$

and the constant $L$ is given by

$$
L:=C^{\prime} \frac{1}{\beta^{p \sigma^{\prime}\left(1-\frac{q \sigma^{\prime}}{\nu^{*}}\right)}}\left(\frac{1}{R_{0}}\right)^{p \sigma^{\prime}} .
$$

We remind that, in its turn, we have

$$
\begin{aligned}
C^{\prime}= & C\left(1+\|u\|_{L^{\nu^{*}\left(B\left(x_{0}, R_{0}\right)\right)}}^{(q-p) \sigma^{\prime}}\right) \\
=c & \left\{\left\|\lambda^{-1}\right\|_{L^{r}\left(B\left(x_{0}, R_{0}\right)\right)} \cdot\left(1+\|a\|_{L^{\tau}\left(B\left(x_{0}, R_{0}\right)\right)}\right) \cdot\left(1+\|\mu\|_{L^{\sigma}\left(B\left(x_{0}, R_{0}\right)\right)}\right)\right\}^{\sigma^{\prime}} \times \\
& \times\left(1+\|u\|_{L^{\left.\left.\nu^{*}(B) \sigma_{0}, R_{0}\right)\right)}}^{(q-p)}\right),
\end{aligned}
$$

where $c>0$ only depends on $n, p, q, r, \sigma, \tau$ and on $k$ and $Q$. We now claim that it is possible to choose $\beta \geq 1$ in such a way that $J_{0} \leq L^{-1 / \alpha}\left(8^{p \sigma^{\prime 2}}\right)^{-1 / \alpha^{2}}$, that is,

$$
\begin{aligned}
& J_{0}=\int_{A_{\frac{\beta}{2}, R_{0}}}\left(u-\frac{\beta}{2}\right)^{p \sigma^{\prime}} \mathrm{d} x \\
& \quad \leq\left\{C\left(1+\|u\|_{L^{\nu^{*}}\left(B\left(x_{0}, R_{0}\right)\right)}^{(q-p) \sigma^{\prime}}\right) \cdot \frac{1}{\beta^{p \sigma^{\prime}\left(1-\frac{q \sigma^{\prime}}{\nu^{*}}\right)}}\left(\frac{1}{R_{0}}\right)^{p \sigma^{\prime}}\right\}^{-1 / \alpha}\left(8^{\left.p \sigma^{\prime 2}\right)^{-1 / \alpha^{2}} .}\right.
\end{aligned}
$$

In fact, by definition of $J_{0}$ (and since $u \in L_{\text {loc }}^{p \sigma^{\prime}}(\Omega)$, see Remark 2.4), we have

$$
J_{0} \leq \int_{B\left(x_{0}, R_{0}\right)}|u|^{p \sigma^{\prime}} \mathrm{d} x=\|u\|_{L^{p \sigma^{\prime}\left(B\left(x_{0}, R_{0}\right)\right)}}^{p \sigma^{\prime}}<\infty
$$


thus, reminding that $R_{0} \leq 1$, the claimed (4.15) is clearly fulfilled if we choose

$$
\beta:=\left\{1+\frac{C}{R_{0}^{p \sigma^{\prime}}}\left(8^{p \sigma^{\prime 2}}\right)^{1 / \alpha}\left(1+\|u\|_{L^{\nu^{*}}\left(B\left(x_{0}, R_{0}\right)\right)}^{(q-p) \sigma^{\prime}}\right) \cdot\|u\|_{L^{p \sigma^{\prime}\left(B\left(x_{0}, R_{0}\right)\right)}}^{p \sigma^{\prime} \alpha}\right\}^{\frac{1}{p \sigma^{\prime}\left(1-\frac{q \sigma^{\prime}}{\nu^{*}}\right)}} .
$$

With (4.15) at hand and $\beta \geq 1$ as in (4.16), we are entitled to apply Lemma 4.3. As a consequence, we obtain

$$
\lim _{h \rightarrow \infty} J_{h}=\lim _{h \rightarrow \infty} \int_{A_{\beta_{h}, \rho_{h}}}\left(u-\beta_{h}\right)^{p \sigma^{\prime}} \mathrm{d} x=\int_{A_{\beta, R_{0} / 2}}(u-\beta)^{p \sigma^{\prime}} \mathrm{d} x=0 .
$$

Since, by definition, $u-\beta>0$ on $A_{\beta, R_{0} / 2}$, from (4.17) we then conclude that

$$
\left|A_{\beta, R_{0} / 2}\right|=0, \quad \text { whence } \quad u \leq \beta \text { for a.e. } x \in B\left(x_{0}, R_{0} / 2\right) \text {. }
$$

To prove that $u$ is also bounded from below by $-\beta$ it suffices to observe that, setting $v:=-u$, then $v \in W_{\text {loc }}^{1,1}(\Omega)$ is a quasi-minimizer of the functional

$$
\mathcal{G}(w):=\int_{\Omega} g(x, w, \nabla w) \mathrm{d} x,
$$

where $g: \Omega \times \mathbb{R} \times \mathbb{R}^{n} \rightarrow \mathbb{R}$ is the function defined by

$$
g(x, w, \xi):=f(x,-w,-\xi) .
$$

As a consequence, since $g$ clearly satisfies assumptions (H1) and (H2) (with the same functions $\lambda, \mu)$, by the above argument we deduce that

$$
\left|A_{\beta, R_{0} / 2}(v)\right|=0, \quad \text { whence } \quad-u \leq \beta \text { for a.e. } x \in B\left(x_{0}, R_{0} / 2\right) \text {. }
$$

Summing up, we have proved that, if $\beta \geq 1$ is as in (4.16), one has

$$
|u(x)| \leq \beta, \quad \text { for a.e. } x \in B\left(x_{0}, R_{0} / 2\right) .
$$

We now observe that, by Hölder's inequality (since $p \sigma^{\prime}<\nu^{*}$ ), we have

$$
\left(1+\|u\|_{L^{\nu^{*}}\left(B_{R_{0}}\right)}^{(q-p) \sigma^{\prime}}\right)\|u\|_{L^{p \sigma^{\prime}\left(B\left(x_{0}, R_{0}\right)\right)}}^{p \sigma^{\prime}} \leq c\left(1+\|u\|_{L^{\nu^{*}\left(B_{R_{0}}\right)}}\right)^{\sigma^{\prime}(q-p+p \alpha)},
$$

with $c>0$ independent of $u$. This, together with (4.16), $R_{0} \leq 1, \varrho^{1 / \alpha} \geq 1$, allows us to conclude that there exists a constant $c>0$, depending on $n, p, q, r, \sigma, \tau$ and on $k$ and $Q$, such that (here, $H$ is as in (2.14))

$$
\beta \leq c\left(\frac{H}{R_{0}}\right)^{\frac{\nu^{*}}{\nu^{*}-q \sigma^{\prime}}}\left(1+\|u\|_{L^{\nu^{*}}\left(B_{R_{0}}\right)}\right)^{\frac{q-p+p \alpha}{p\left(1-\frac{q \sigma^{\prime}}{\nu^{*}}\right)}} .
$$

This inequality and (4.18) give exactly estimate (2.13).

\section{REFERENCES}

[1] P. Bella, M. Schäffner: Local boundedness and Harnack inequality for solutions of linear nonuniformly elliptic equations, accepted on Comm. Pure App. Math. (2019).

[2] P. Baroni, M. Colombo, G. Mingione: Regularity for general functionals with double phase, Calc. Var. Partial Differential Equations 57 (2018), no. 2, Art. 62, 48 pp.

[3] M. Colombo, G. Mingione: Regularity for double phase variational problems, Arch. Rat. Mech. Anal. 215 (2015), 443-496.

[4] M. Colombo, G. Mingione: Bounded minimisers of double phase variational integrals, Arch. Rat. Mech. Anal. 218 (2015), 219-273.

[5] D. Cruz-Uribe, P. Di Gironimo, C. Sbordone: On the continuity of solutions to degenerate elliptic equations, J. Differential Equations 250 (2011), 2671-2686.

[6] G. Cupini, P. Marcellini, E. Mascolo: Regularity of minimizers under limit growth conditions, Nonlinear Anal. 153 (2017), 294-310.

[7] G. Cupini, P. Marcellini, E. Mascolo: Nonuniformly elliptic energy integrals with p,q-growth, Nonlinear Anal. 177 (2018), part A, 312-324.

[8] E. De Giorgi: Sulla differenziabilità e l'analiticità delle estremali degli integrali multipli regolari, Mem. Accad. Sci. Torino. Cl. Sci. Fis. Mat. Nat. 3 (1957) ,25-43. 
[9] G. Di Fazio, M.S. Fanciullo, P. Zamboni: Local regularity for strongly degenerate elliptic equations and weighted sum operators, Diff. Integral Eq. 32 (2019), 479-492.

[10] E. Fabes, C. Kenig, R. Serapioni: The local regularity of solutions of degenerate elliptic equations, Comm. Partial Diff. Eq. 7 (1982), 77-116.

[11] M. Giaquinta, E. Giusti: On the regularity of the minima of variational integrals, Acta Math. 148 (1982), 31-46.

[12] M. Giaquinta, E. Giusti: Differentiability of minima of nondifferentiable functionals, Invent. Math. 72, no. 2 (1983), 285-298.

[13] M. Giaquinta, E. Giusti: Quasiminima, Ann. Inst. H. Poincaré Anal. Non Linéaire 1, no. 2 (1984), 79-107.

[14] E. Giusti: Direct methods in the Calculus of Variations, World Scientific Publishing Co., Inc., River Edge, NJ (2003).

[15] T. Iwaniec, L. Migliaccio, G. Moscariello, A. Passarelli di Napoli: A priori estimates for nonlinear elliptic complexes, Adv. Differential Equations 8 (2003), 51-546.

[16] P. Marcellini: Regularity and existence of solutions of elliptic equations with $p-q$-growth conditions, J. Differential Equations 90 (1991), 1-30.

[17] P. Marcellini, C. Sbordone: Homogenization of nonuniformly elliptic operators, Applicable Anal. 8 (1978/79), 101-113.

[18] L. Migliaccio, G. Moscariello: Higher integrability of div-curl products, Ricerche Mat. 49 (2000), 151-161.

[19] G. Moscariello: Local boundedness of minimizers of certain degenerate functionals of the calculus of variations, Nonlinear Anal. 23 (1994), 1587-1593.

[20] J. Moser: On Harnack's theorem for elliptic differential equations, Comm. Pure Appl. Math. 14 (1961), 577-591.

[21] B. Muckenhoupt: Weighted norm inequalities for the Hardy maximal function, Trans. Amer. Math. Soc. 165, 1972, 207-226.

[22] G. Stampacchia: Equations elliptiques du second ordre à coefficients discontinus, (French) Séminaire de Mathématiques Supérieures, No. 16 (Été, 1965) Les Presses de l'Université de Montréal, Montreal, Que. 1966.

[23] N.S. Trudinger: On the regularity of generalized solutions of linear, non-uniformly elliptic equations, Arch. Rational Mech. Anal. 42 (1971), 42-50.

[24] N.S. Trudinger: Linear elliptic operators with measurable coefficients, Ann. Scuola Norm. Sup. Pisa Cl. Sci. (3) 27 (1973), 265-308.

[25] X. Zhong: Discontinuous solutions of linear degenerate elliptic equations, J. Math. Pures Appl. (9) 90 (2008), 31-41.

(Stefano Biagi) Dipartimento di Ingegneria Industriale E Scienze Matematiche Università Politecnica della Marche Via Brecce Bianche, 60131, Ancona, Italy

E-mail address: s.biagi@dipmat.univpm.it

(Giovanni Cupini) Dipartimento di MatematicA

Università degli Studi di Bologna

Piazza di Porta SAn Donato 5, 40126, Bologna, Italy

E-mail address: giovanni.cupini@unibo.it

(Elvira Mascolo) Dipartimento di Matematica e Informatica "U. Dini"

UNiversità DEGLi STUdi di FiREnZE

Viale Morgagni 67/A, 50134, Firenze, Italy

E-mail address: elvira.mascolo@unifi.it 